\title{
MÁQUINAS ELÉCTRICAS UNIPOLARES
}

\author{
Jorge Guala V. ${ }^{1} \quad$ Pedro Mazzoni $^{1}$
}

Recibido el 16 de enero de 2002, aceptado el 06 de agosto de 2002

\section{RESUMEN}

Describimos dos experimentos, cuasi triviales pero indiscutibles, que desaprueban las interpretaciones absolutistas del tipo Kennard. Nuestros resultados están de acuerdo con lo establecido por Weber en lo concerniente al movimiento relativo en electrodinámica. También concordamos en lo concerniente a movimiento a nivel general con la visión de Mach. Los resultados reportados aquí pueden motivar a los ingenieros a comenzar a diseñar una nueva generación de motores unipolares sin escobillas.

Palabras Claves: Electrodinámica, relatividad, interconversión de energías mecánica-

\begin{abstract}
Two quasi trivial but undisputable experiments, which disprove Kennard-type absolutistic interpretations of unipolar machines, are discussed. Findings are in agreement with Weber's statements concerning the role of relative motion in electrodynamics. Agreement is also found with Mach's views, concerning motion at the most general level. The findings here reported should encourage engineers to start the design of a new generation of "brushless" unipolar engines.
\end{abstract}

Keywords: Electrodynamics, relativity, interconversion of energy electric-mechanical

\section{RESUMEN}

La generación y aprovechamiento de las corrientes eléctricas han permitido cambiar, de una manera radical, las condiciones de vida de la Humanidad. Recurriendo a una frase que, aunque vulgar y manida, rebosa de contenido, diremos, una vez más, que hay un antes y un después de la electricidad.

La fascinante historia de la ciencia eléctrica cobra singular atractivo con el descubrimiento, fortuito por cierto, de Michael Faraday en 1831. Nos estamos refiriendo al fenómeno de inducción electromagnética, alma de nuestros modernos generadores y motores.

Físicos e ingenieros se valen hoy de la ley de inducción de Faraday para comprender y diseñar nuevas y cada vez más eficientes máquinas generadoras, ya de corriente, ya de fuerza. La inmensa mayoría de estas máquinas se valen de las variaciones que, en el transcurso del tiempo, experimenta el campo magnético sobre alambres capaces de conducir corriente.

Hay, sin embargo, otra categoría de ingenios que son capaces de producir corrientes y fuerzas motrices, sin que sobre ninguna de sus piezas constituyentes se opere la menor variación del vector magnético. Son estas las poco conocidas máquinas unipolares, de las que el Disco de Faraday es el primer ejemplo que nos presenta la Historia.

El funcionamiento de estas máquinas se convirtió en un verdadero misterio por casi 170 años, como fácilmente puede comprobarse consultando la literatura internacional. El problema central en el tema que nos ocupa es la localización espacial del asiento de los fenómenos de generación de corrientes y de fuerzas.

\section{ELDINAMOTOR}

Hacia 1832 Faraday desarrolló un generador de corriente continua muy simple, conocido posteriormente con el nombre de Disco de Faraday (Fig. 1)

Esencialmente consta de un disco metálico, $(D)$, capaz de rotar en las proximidades del polo de un imán permanente $(M)$. Cuando los extremos de un alambre

\footnotetext{
1 Universidad Tec.nológica Nacional, U. Académica Plaza Huincul, Neuquén, Argentina, fundacionjuliopalacios@usa.net,micromec@ciudad.com.ar
} 
conductor, que denominaremos en adelante circuito de cierre, $(C C)$, se apoyan, respectivamente, sobre el eje y la periferia del disco, una corriente continua fluye a través del $C C$. Invirtiendo el sentido de la rotación, la corriente generada cambia su sentido. Otro tanto ocurre al cambiar el polo del imán próximo al disco.

La totalidad de los hechos observados se explica sin más que recurrir a la ley de fuerza de Lorentz, $f_{L}=q(v x B)$, expresión en la que $v$ representa el vector velocidad $(\mathrm{m} / \mathrm{s})$ con que cualquier carga eléctrica $q$ (culombios) de $D$ se desplaza, en virtud del movimiento de rotación, en una región del espacio en la que existe un campo magnético $\boldsymbol{B}\left(\mathrm{Wb} / \mathrm{m}^{2}\right)$.

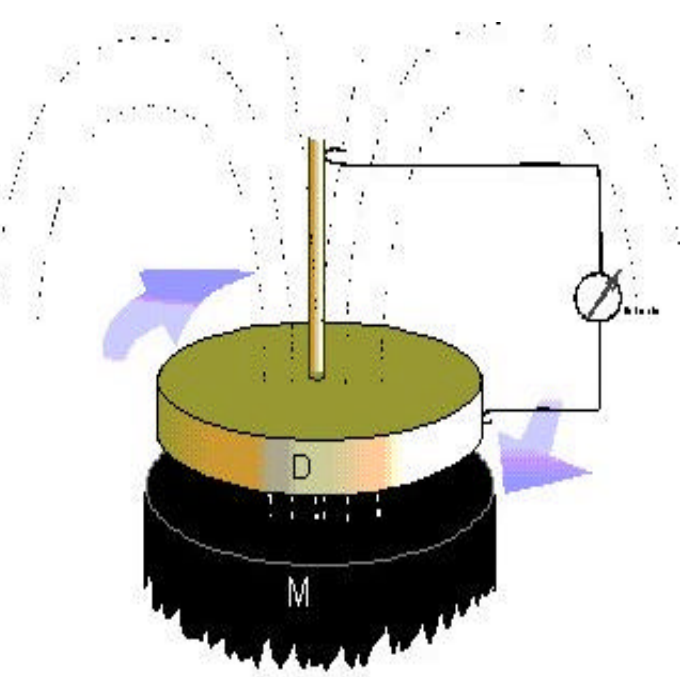

Fig. 1.- Disco de Faraday

La Fig. 2 muestra la vista superior de $D$ rotando en sentido anti-horario (SAH) sobre el polo norte de $M$. El símbolo - (punta de flecha) representa al vector $\boldsymbol{B}$, saliente del plano de la figura. La fuerza de Lorentz arrastra las cargas eléctricas positivas hacia la periferia del disco, que resulta eléctricamente polarizado. Sabemos que, en realidad, lo que se mueve es la carga eléctrica negativa (electrones) hacia el centro de $D$.

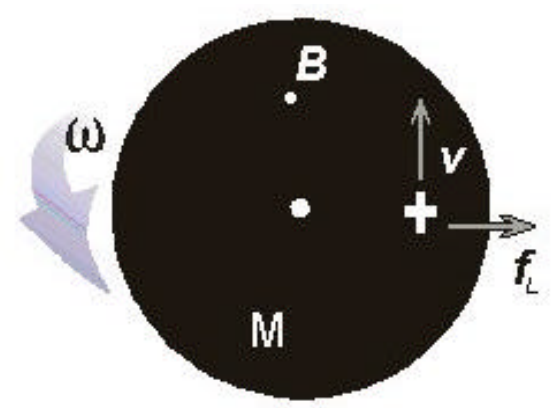

Fig. 2.- Vista superior del disco de rotación
El disco así polarizado se comporta como un generador de fuerza electromotríz (fem). Integrando $\boldsymbol{v} x \boldsymbol{B}$ desde el centro del disco hasta un radio arbitrario $r$ se calcula fácilmente la cuantía, $\boldsymbol{\varepsilon}$, de dicha $\mathrm{fem}$, resultando valer $\varepsilon=\omega \mathrm{Br}^{2} / 2$ (voltios), donde $\omega$ simboliza la velocidad angular de rotación $(\mathrm{rad} / \mathrm{s})$.

Mediante una adecuada elección topológica es posible construir un generador unipolar "quasi-lineal" [1], en el que la fem depende linealmente de la distancia.

Un fenómeno llamativo ocurre cuando el imán se suelda al disco y ambos se ponen en rotación. Las mediciones muestran exactamente los mismos valores que en el caso anterior. La generación de corriente, tanto en intensidad como en sentido, ocurre como si $M$ no participase del movimiento. Este simple hecho observado admite dos interpretaciones, irreconciliables entre sí, que dieron lugar al establecimiento de otras tantas escuelas en las que los científicos se repartieron a lo largo de 170 años: la absolutista y la relativista.

Para un absolutista (A) el fenómeno unipolar está gobernado por la rotación absoluta de $D$. Toda rotación de $M$ es irrelevante en lo que hace a la polarización de $D$ $\mathrm{y}$, por consiguiente, a la generación de corriente. Téngase presente que, por simetría, al ser de distribución de $\boldsymbol{B}$ en el espacio que circunda a $M$, será idénticamente $\partial \boldsymbol{B} / \partial t=0$ en todos y cada uno de los puntos de $D$. Recordemos que $\partial \boldsymbol{B} / \partial t \neq 0$ es la condición ineludible para la generación de fem en los generadores ordinarios. Esta condición es la que hace aplicable la conocida ley de inducción de FaradayHenry.

Para un relativista $(\mathbf{R})$ el fenómeno está gobernado por la rotación relativa entre $M$ y los conductores. Dado que $M$ y $D$ se encuentran en reposo relativo cuando ambos rotan conjuntamente, entonces $\mathbf{R}$ debe buscar en el $C C$ la localización del origen de la fem observada.

La Fig. 3 ilustra elocuentemente los dos puntos de vista arriba expuestos. Nótese que la polarización de $D$ tiene el mismo sentido que el de la Fig. 2. Esto significa que, para $\mathbf{R}$, tanto da rotar $D$ en $\mathrm{SAH}$ como rotar $M$ en sentido horario $(\mathrm{SH})$.

La Fig. 3 sólo nos refiere un sistema de "dos cuerpos", $D$ y $M$ y las complicaciones aparecen en el momento de introducir el $C C$ con el fin de obtener corriente. Este circuito de cierre, al permanecer estacionario en el laboratorio, no juega, para $\mathbf{A}$, ningún rol activo en lo que a generación concierne. Su papel, enteramente pasivo, se reduce al de proveer un camino conveniente para el flujo de la electricidad. 


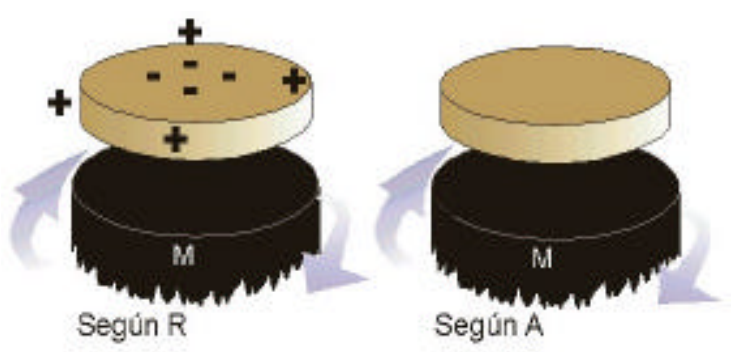

movimientos relativos entre las partes relevantes involucradas [2]. Más de medio siglo más tarde [3] Einstein enfatizó la importancia del punto de vista de Weber, en su famoso artículo de 1905, habitualmente reputado como la génesis de la teoría de la relatividad.

Kennard merece ser citado como conspicuo exponente de la escuela absolutista [4], [5].

Por extraño que parezca, durante los 170 años que nos separan desde Faraday hasta hoy, coexistieron las dos escuelas antagónicas, debido a la falta de experimentos irrefutables, capaces de validar una de ellas, descartando por consiguiente la opuesta.

\section{ROTOR ASIMÉTRICO}

La Fig. 4 muestra un imán cerámico al que se le ha practicado un corte que representa cerca de 1/30 de su superficie. El símbolo $\times($ cola de flecha) representa al vector campo magnético, $\boldsymbol{B}$ entrante. Nótese que en el corte tiene lugar la inversión de las líneas de campo. En lenguaje de Faraday, diríamos que el campo "prefiere cerrarse" radialmente sobre $M$ antes que por la periferia del disco. Esta asimetría se manifiesta con intensidad sólo en las inmediaciones del corte y no perturba sensiblemente la distribución global de $\boldsymbol{B}$ entorno del imán (Fig. 5).

El imán, (radio interno $25 \mathrm{~mm}$, externo $75 \mathrm{~mm}$ ) está empotrado en un disco de teflón de 20 centímetros de diámetro. Los alambres conductores ap y $q r$ están simétricamente soldados al disco (Fig. 4). Una pista circular de cobre, $c$, permite el contacto eléctrico entre ambas ramas. El extremo exterior de $a b$ está soldado a un anillo circular de cobre, $C_{a}$, anclado en las proximidades del borde superior del disco. Un alambre conductor $r b$ posibilita el contacto eléctrico entre $q$ y un segundo anillo de cobre, $C_{b}$, anclado al disco, unos $4 \mathrm{~mm}$ por debajo de $C_{a}$.

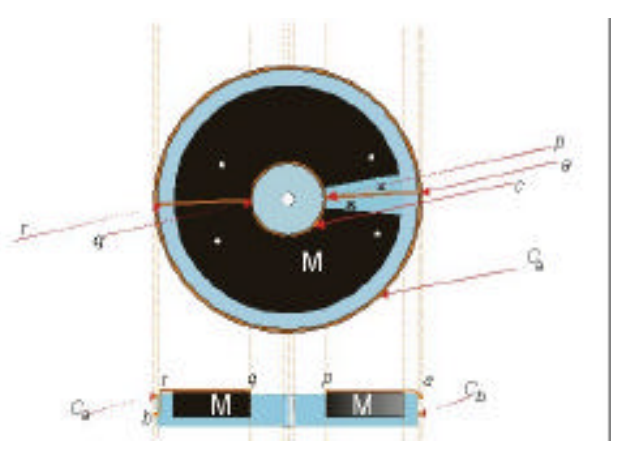

Fig. 4.- Imán cerámico con corte

Tenemos así un generador unipolar que consta de cinco piezas, a saber: $M$, los conductores $a b, p q, q r$, y el $C C$. Denominaremos rotor, $R$, al conjunto constituido por las cuatro primeras piezas. $R$ fue dinámicamente balanceado, para corregir la pérdida de masa de $M$, debida al corte.

La peculiar topología adoptada excluye la posibilidad de generación de importantes pulsos de corriente alterna ( a la frecuencia de rotación), debida a una variación temporal del flujo de $\boldsymbol{B}$ dentro de la superficie encerrada por los conductores. Como una prueba adicional de lo antes dicho se envolvió, con una bobina de alambre de 600 vueltas, en forma simétrica, el rotor $R$. Con la ayuda del osciloscopio se midió una tensión, pico a pico, a la frecuencia de rotación, menor que $50 \mathrm{mV}$, cuando la rotación alcanzó las $1.500 \mathrm{rpm}$. Esto corresponde a una señal inferior a los 0,1mV/espira, completamente despreciable para el fenómeno estudiado.

Lo que sí resulta inevitable, es el ruido aleatorio generado por los contactos deslizantes. Para emover estas señales espurias, se conectó en paralelo con el $C C$ un capacitor de unos $200 \mu \mathrm{F}$.

\section{Experimental 1: Generador}

\section{G-1}

Permaneciendo $R$ firmemente anclado al banco de trabajo, se puso en rotación, centrado sobre $R$ con una separación de $5 \mathrm{~mm}$, un disco homogéneo de aluminio (80 $\mathrm{mm}$ de radio). Seguidamente se procedió a medir tensión (diferencia de potencial) entre los puntos $a p, q r$, 
ar correspondientes a $R$. Al alcanzar unas $1.500 \mathrm{rpm}$ (ca. $150 \mathrm{rad} / \mathrm{s}$ ), en $\mathrm{SH}$, se obtuvo:

$V_{a p}=V_{a}-V_{p}=+2,0 \pm 0,1 \mathrm{mV} ; V_{q r}=V_{q}-V_{r}=$ $+20,0 \pm 0,1 \mathrm{mV} ; V_{a r}=22,0 \pm 0,1 \mathrm{mV}$

No ha de extrañar el pequeño valor de la tensión medida entre los puntos ap sobre el disco en rotación, dado que al ingresar el mismo en la zona de inversión del campo, se engendran corrientes de Foucault que reducen la diferencia de potencial debida al efecto unipolar puro. Estas corrientes parásitas pueden disminuirse ranurando radialmente el disco. Esto no representa ninguna molestia para nuestros propósitos actuales. Lo que es relevante, en este punto, es reconocer que el disco en rotación se comporta como un acoplamiento en serie de dos generadores independientes, polarizados en forma opuesta. Nótese que sobre la rama derecha ( $a p)$ la carga positiva se acumula en la periferia del disco, en tanto que sobre la izquierda ocurre lo contrario. Todo esto es previsible, naturalmente, a partir de la aplicación de la ley de fuerzas de Lorentz.

\section{G2}

Sustituyendo el disco por un anillo de bronce de $30 \mathrm{~mm}$ de altura, capaz de rotar centrado en $R$, separado unos $3 \mathrm{~mm}$ de su periferia se procedió a medir la tensión correspondiente a los puntos $r b$ de $R$. Así se obtuvo, $V_{r b}=0,0 \pm 0,1 \mathrm{mV}$.

Dejando a un lado disco y anillo, se procedió a medir tensión, en los puntos de interés, $C_{a}, C_{b}, c$, sobre $R$ en rotación (SH). Se aumentó gradualmente la velocidad de rotación hasta alcanzar, midiendo entre $c$ y $C_{b}$, la tensión de $20 \mathrm{mV}$. Las mediciones arrojaron los valores: $V_{a p}{ }^{\prime}=\underline{-20} \pm \underline{0,1 m V} ; V_{q b^{\prime}} \approx V_{q r}{ }^{\prime}=+20,0 \pm 0,1 \mathrm{mV}$ $V_{a b}{ }^{\prime}=0,0 \pm 0,1 \mathrm{mV}$.

\section{Análisis}

Dado que para A el fenómeno básico de inducción está localizado en las ramas ap y $q r$ de $R$, él esperaba medir una tensión $V_{a b}{ }^{\prime} \approx V_{a r}>+20 m V$, y $V_{a p}{ }^{\prime}>0$, en lugar de los $-20 m V$ medidos. La primera desigualdad toma cuenta de la ausencia de las corrientes parásitas de Foucault en esta configuración.

En otras palabras, lo que A espera como resultado de estas mediciones, es la tensión correspondiente a un acoplamiento en serie de dos generadores opuestamente polarizados, conectados en serie, al igual que en G-1.

Llegado a este punto, A encuentra severas dificultades ara explicar, con los elementos involucrados, los fenómenos observados. Recordemos que para él, el $C C$ no juega más que un papel pasivo en el mecanismo de generación de fem.

Desde el punto de vista de $\mathbf{R}$, el fenómeno de inducción tiene su asiento en el movimiento relativo entre $M$ y el $C C$. Ninguna de las ramas conductoras en rotación, por hallarse en reposo con relación a, M puede ser fuente de fem. El único rol de las mencionadas ramas es enteramente pasivo en lo que a generación concierne.

o ofrecen un camino apto para el flujo de la corriente.

Lo que realmente interesa a $\mathbf{R}$ en el fenómeno considerado es la rotación de $R$ con relación al $\boldsymbol{C C}$. El corte en el que se aloja la rama $p q$ sólo introduce una perturbación local menor sobre la distribuci del campo $\boldsymbol{B}$ entorno de $M$. Esta perturbación es incapaz de desnaturalizar el efecto inductivo principal, que tiene lugar sobre el $C C$.

Volviendo al lenguaje de Faraday, el $C C$ "ve", esencialmente, la misma distribución de $\boldsymbol{B}$ en el transcurso del tiempo (Fig.5).

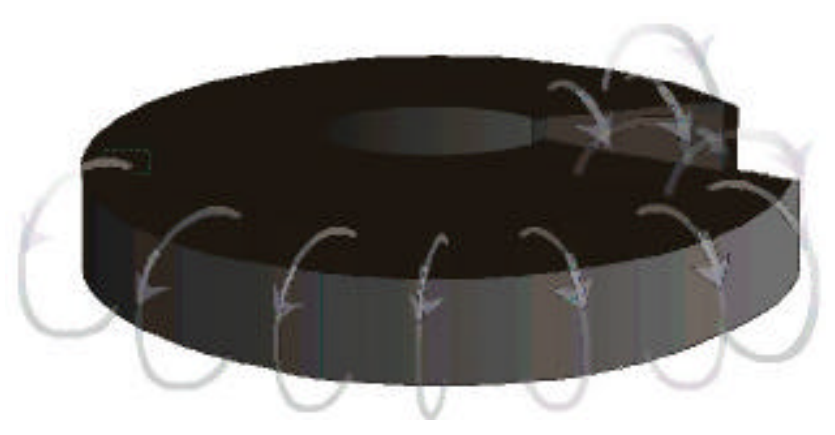

Fig. 5.- Distribución de líneas de campos en el entorno del imán cilíndrico con corte

Como una prueba adicional de lo arriba expuesto, hemos preparado otro imán en el que el corte sólo importaba $1 / 150$ de la pieza completa. Los resultados obtenidos, dependientes de la inversión de $\boldsymbol{B}$ en la singularidad, concordaron con los arriba descriptos. Aquí, como es obvio, la distribución espacial de $\boldsymbol{B}$ permanece esencialmente invariable.

De este modo, $\mathbf{R}$ no se sorprende al medir $V_{a b}{ }^{\prime}=0$, por cuanto está en la situación descrita en la introducción: el

$M$ del rotor $R$ polariza idénticamente los extremos del $C C$; consecuentemente, ninguna corriente puede fluir. 


\section{Experimental 2: Motor}

La Fig. 6 muestra el rotor de los anteriores experimentos ligeramente modificado, de modo tal que resulta conveniente para la investigación de fuerzas ponderomotrices cuando funciona como motor. Ahora el imán está empotrado en un bastidor de madera, provisto de dos canales semicirculares capaces de alojar mercurio. El rotor $R$ está rígidamente soldado a un eje conductor, rematado en aguzadas puntas, capaces de rotar sobre un superficie vítrea pulida. El dispositivo ofrece un bajísimo par (cupla, torque) friccional. Los extremos internos de las ramas $a X, b X$ están soldados al eje, en tanto que los externos, previamente doblados, se sumerjen en su respectivo canal de mercurio.

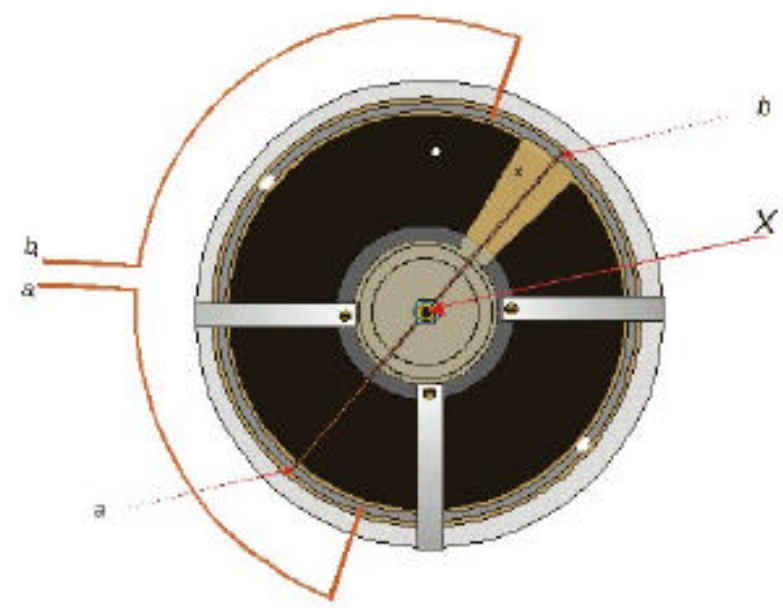

Fig.6.- Rotor del primer experimento modificado

\section{M1}

Se inyecta corriente contínua en el canal $a_{l}$ permitiendo el retorno de la misma a la fuente, mediante el $C C$, a través del eje $X$. En las condiciones experimentales adoptadas, al superar los 2 A (amperes), se observa una neta rotación del rotor $R$ en SAH. La corriente de 2 A es la mínima capaz de asegurar, en estas condiciones, un par capaz de vencer las fuerzas de fricción, principalmente debidas al contacto entre el alambre estacionario y el mercurio. Al invertir el sentido de la corriente, naturalmente, se invierte también el sentido

Desde el punto de vista de $\mathbf{A}$, es la rama $a X$ la que juega el papel activo en la generación de la cupla responsable de la rotación. De este modo, la fuerza de Laplace, $d \boldsymbol{F}=I(d \boldsymbol{l} x \boldsymbol{B})$, integrada a lo largo de la rama en cuestión, provee la fuerza de arrastre que produce la rotación de $R$. Debido a la flexibilidad de la rama $a X$ se aprecia un ostensible encorvamiento de la misma en el
SAH cuando la corriente fluye. El movimiento del alambre coincide con el del disco.

Para R, en cambio, $M$ juega el rol preponderante en la génesis del movimiento de rotación observado. Así, $M$ repele (atrae) a $a X$ en el $\mathrm{SAH}$, pero a la vez atrae (repele) al $C C$ en el SH. El hecho de estar $a X$ soldada a $R$ hace que las fuerzas debidas a la interacción $M-a X n o$ contribuyan a la rotación de $\boldsymbol{R}$ (Tercera Ley del movimiento de Newton). Aún cuando presentes, estas fuerzas son incapaces de generar un par rotacional.

Quedan tan sólo las fuerzas provenientes de la $M-C C$ para explicar el fenómeno observado. Dado que, como vimos, $M$ imprime al $C C$ un par de $\mathrm{SH}$, el circuito de cierre reacciona sobre $R$ engendrando en éste una cupla de $\mathrm{SAH}$, responsable de la rotación observada, nuevamente de acuerdo con el Principio de acción-reacción de Newton.

\section{M-2}

El paso siguiente consistió en inyectar corriente continua en el eje $X$, permitiendo a la misma retornar a la fuente a través de la rama $b X$. Superados los $2 \mathrm{~A}$, el rotor $R$ experimenta una neta rotación de sentido horario, a pesar de que la rama $b X$ experimenta una ostensible deformación en SHA, como es de esperar por simple aplicación de la ley de Laplace. Alambre y disco adquieren ahora movimientos opuestos.

\section{Análisis}

El resultado de este experimento resulta difícil, si es que posible, de explicar en términos de la concepción de $\mathbf{A}$, basada, como vimos, en la hipótesis del "arrastre" de $M$ por parte de $b X$.

Por el contrario, $\mathbf{R}$ no encuentra dificultad alguna en reconocer que, aunque contrarias a las del experimento M-1, las fuerzas de interacción entre $M$ y $b X$ no pueden engendrar rotación alguna, por el simple hecho de estar simultáneamente aplicadas al mismo cuerpo $R$ genera sendos pares, en sentido horario, sobre $b X$ y sobre el circuito de cierre, $C C$. Nótese que, al actuar M sobre el CC, todo pasa como si no existiese la singularidad (corte). El circuito de cierre reacciona sobre $M$ poniendo a $R$ en rotación de sentido horario.

\section{M-3}

Finalmente, inyectamos corriente en $a_{1}$, permitiendo su retorno a la fuente por $b_{1}$. Al superar los $2 \mathrm{~A}$ se observa un franco encorvamiento de los alambres $a X, b X$, ambos en el $S A H$. El rotor $R$, sin embargo, no muestra la menor señal de rotación, aún cuando la corriente fue 
llevada hasta los 100 A (con el consiguiente calentamiento de los alambres y del mercurio).

\section{Análisis}

De acuerdo con A los alambres aX y $b X$, sometidos a sendas fuerzas capaces de generar pares de SAH sobre los mismos, deberían poner al rotor $R$ en rotación del mismo sentido. Encuentra, naturalmente, serias dificultades para explicar el fenómeno observado.

Para $\mathbf{R}$, en cambio, la explicación es por demás sencilla. Cuando trata de identificar al circuito de cierre, $C C$, sólo encuentra segmentos circulares de ambos canales de mercurio y también segmentos circulares de alambres que conectan aquellos con la fuente (Fig. 6). Por ser la fuerza de Laplace siempre perpendicular al elemento de corriente considerado, su recta de aplicación pasará, en todos los casos, por el eje $X$, con lo que no podrá esperarse ningún par rotacional, generado por $M$, sobre el $C C$. En virtud de la Tercera ley de Newton, tampoco podrá el $C C$ engendrar ningún par rotacional sobre $M$. Estas últimas consideraciones se pueden fácilmente generalizar para circuitos de cierre de forma arbitraria. Bastan para ello sencillas consideraciones topológicas, la ley de Laplace y la propiedad fundamental del campo, $\operatorname{div} \boldsymbol{B}=0$. Esta última resume el hecho de ser las líneas del campo magnético cerradas (no tienen principio ni fin).

En términos llanos, $\mathbf{R}$ encuentra que, en la configuración descripta, no existe circuito de cierre activo para la generación de fuerzas ponderomotrices sobre $\mathbf{R}$

La Fig. 7 ilustra la interacción entre el imán y el circuito de cierre. La corriente, que proviene del polo positivo de la fuente (+), ingresa en el canal de mercurio, luego pasa al $C C$, retornando al polo negativo (-) de la fuente, tras recorrer el alambre horizontal que reposa sobre $M$ e ingresar en el respectivo canal de mercurio.

Asumiendo que estamos trabajando sobre el polo Norte de $M$, las fuerzas relevantes involucradas son provistas por: $M$ sobre el alambre horizontal, responsable de una cupla (no representada en la figura) de SH sobre el mismo.

Alambre horizontal sobre $M$. Una fuerza igual $y$ contraria a la anterior, responsable de un par de SAH (no representado en la figura) sobre $M$.

$M$ sobre el $C C$, responsable de una cupla de $\mathrm{SAH}$ (representada por una flecha en la figura). Sobre el circuito de cierre.
Circuito de cierre sobre $M$. Una fuerza igual y contraria a la anterior es la responsable de una cupla capaz de mover en imán en el SH.

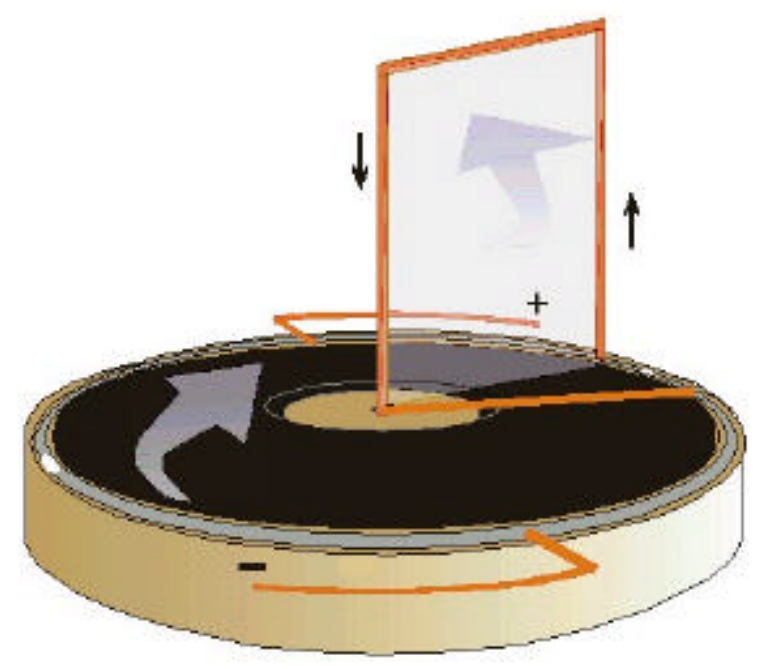

Fig. 7.- Interacción entre el imán y el circuito de cierre

\section{CONCLUSIONES}

Los experimentos descriptos permiten afirmar, sin género alguno de duda, que el fenómeno unipolar, que constituyó un verdadero enigma por el lapso de casi dos siglos, está gobernado por el movimiento relativo entre imán y conductor. Este último puede, según sea la configuración adoptada, ser el rotor o el circuito de cierre.

La importancia de la presente investigación está anclada en dos aspectos:

- Uno, de naturaleza meramente utilitarista, se resume destacando que los ingenieros podrán, al conocer el asiento de los fenómenos electromotrices y ponderomotrices unipolares, aplicar racionalmente su técnica y su arte para el diseño racional y eficiente de nuevas máquinas. No está demás recordar el estrepitoso fracaso que exhibieron, no tan lejos en el tiempo, los cañones electromagnéticos ("railguns" de la guerra de las galaxias). Sus diseñadores ignoraron la localización de las fuerzas de reacción, con lo que los rieles que, además de conducir corrientes enormes, alojaban el proyectil, se deformaban al ser disparados [6] Este sólo hecho convirtió en perfectamente inútil un artificio que mucho prometía (las velocidades adquiridas por los proyectiles son mucho mayores que las obtenidas convencionalmente, mediante deflagración de productos químicos). 
- $\quad$ El otro, que para nada se ocupa de las aplicaciones concretas del conocimiento, viene a llenar una de las más exigentes aspiraciones del humano. Tal es aprender de la maraña de fenómenos que, a diario, lo impactan, gratificándolo o molestándolo, sus elementos centrales, descartando, tanto como le sea posible, la superflua y efímera hojarasca.

- " En la ciencia, como en los planos más profundos $y$ vitales de la cultura, ai posteri l'ardua sentenza". Julio Rey Pastor [7].

\section{REFERENCIAS}

[1] J. Guala-Valverde and P. Mazzoni; "American Journal of Physics" Vol. 63, pp. 228, 1995, 64, 1996, Apeiron 8, 41, 2001.

[2] A.K.T. Assis; "Weber Electrodynamics", Kluwer, Dordrecht, 1994.

[3] A. Einstein; "El Significado de la Relatividad", añola de Carlos Prelat, Espasa Calpe, Madrid, 1971. Título de la obra original: "The Meaning of Relativity", Princeton University Pres s, 1922, 1945, 1950, 1953, 1956.

[4] E. H. Kennard; Philos. Magazine Vol. 23, pp. 937 1912; Vol. 33, pp. 179, 1917.

[5] Bartlett D. F. et al.; "Physical Review” D Vol. 16, pp. 3459, 1977.

[6] P. \& N. Graneau; "Physical Review", E Vol. 63, pp. 058601, 2001.

[7] J. Rey Pastor, P.Pi Calleja, C.A. Trejo; "Análisis Matemático", Vol. I, II, III., Kapelusz, Buenos Aires, 1965. 